\title{
Wireless Remote Patient Monitoring System: Effects of Interference
}

\author{
Andrew Yearp, David Newell, Philip Davies, Russell Wade, Reza Sahandi \\ Faculty of Science \& Technology \\ Bournemouth University \\ Bournemouth, UK \\ ayearp@bournemouth.ac.uk,dnewell@bournemouth.ac.uk \\ daviesp@bournemouth.ac.uk, rwade@bournemouth.ac.uk, \\ rsahandi@bournemouth.ac.uk
}

\begin{abstract}
Wireless remote patient monitoring has the scope to improve patient experience while enabling data transmission to support effective patient care. Dash7 and ZigBee are potential wireless technologies which can facilitate this. Dash7 or ZigBee, when integrated with a mobile phone accompanied by the patient, can overcome the problem of range limitation imposed by traditional wireless patient monitoring solutions. A wireless remote monitoring system using integrated Dash7 or ZigBee and mobile phone technologies is proposed. Reliability is a key requirement in patient monitoring systems. Signal interference can affect the reliability of wireless systems. This paper investigates signal interference on Dash7 and ZigBee in a multitechnology environment. We further consider some of the the ethical implications of using Context Aware systems and their impact upon privacy and show that such tracking systems are ethically feasible but may require additional patient consent.
\end{abstract}

Keywords - Ubiquitous Computing, Wireless Remote Patient Monitoring, Wi-Fi, Interference, Bluetooth, ZigBee, Dash7

\section{INTRODUCTION}

Wireless remote patient monitoring (WRPM) is increasing in use across the healthcare industry, particularly in the United States. However several challenges are facing this integration, including congestion, interference, coexistence issues, fast response, processing of the reported health-related data, supporting the maximum possible number of users and flexibility in operation [1].

Interference from coexistent systems affects reliability, which is a priority in medical applications. The demand for Wi-Fi access has grown quickly and there are a large number of 802.11 devices operating. In such congested environments, with a high density of simultaneous Wi-Fi users, 802.11 devices can cause interference with medical sensors, resulting in a failure to alert physicians to urgent situations [2].

This paper investigates interference in a co-existent technological environment of ZigBee and Dash7 sensors by Bluetooth and Wi-Fi signals and by one another.

\section{TECHNOLOGY REVIEW}

Commonly used wired monitoring systems affect patient mobility and comfort. The lack of mobility caused by wired monitoring systems is discussed by Baisa [3]. Although some wireless monitoring systems have been proposed $[4,5]$, they are being introduced in hospital wards which potentially impact on a number of issues including privacy.

WRPM sensors need to be battery powered whilst keeping power usage to a minimum, this ensures a sufficient operating time. The sensors must provide an adequate range within a built environment however power consumption normally increases when extending the range of a transmission, thus limiting the types of wireless technology that can be used. Bluetooth and ZigBee can be considered suitable for this application, although other technologies do exist e.g. cellular [6].

Many wireless technologies, including WiFi and ZigBee, operate in the crowded 2.4GHz ISM (Industrial, Scientific and Medical) bands. Dash7 however operates at the lower $433 \mathrm{MHz}$ band with advertised read ranges of 1.5 kilometer or more, although ranges of up to $10 \mathrm{~km}$ have been tested by Savi Technology[7]. Therefore Dash7 is thought to be unaffected by interference from the higher frequency ISM band but it may be affected by sideband interference from other devices.

Whereas ZigBee has been incorporated into many patient monitoring systems, Dash7 [8] has normally been used for location-based features in devices such as smartcards, key fobs, tickets, watches and other domestic products. Dash7 is being considered here for its possible interest in medical applications because its use of the lower frequency band, expected lower levels of interference and possible benefits for a WRPM system. As shown in Fig 1[9], Dash7 has the advantage of being low power, long range, and low cost, as well as using the less-crowded low frequency band. This is in contrast to the relatively higher-power wireless technologies like Wi-Fi or Bluetooth.

\section{WPRM DESIGN PROPOSAL}

A fully operation WPRM monitoring system requires not only the use of short range sensor technology such as ZigBee or Dash7 but it also requires a mechanism to transmit that data to a remote database. We propose a system architecture in which sensors transmit vital signs from a patient via a mobile phone as a local base-station which sends the data to a network-based server for data storage. Fig 2 illustrates the system which can employ either ZigBee or Dash7 as patient sensors.

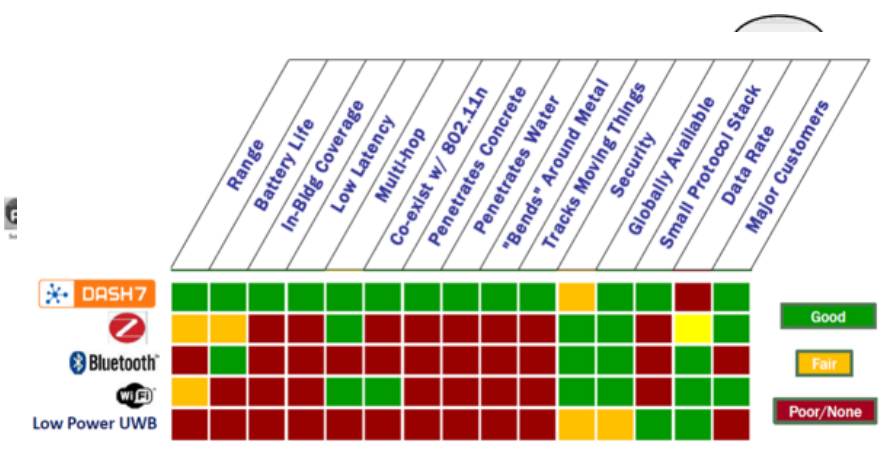

Fig. 1. Wireless comparison Fig 2 System Design 
This system picks up data from sensors on the patient and communicates that date onto the ZigBee or Dash7 network. The data is then passed to a mobile phone which patients keep close to them, either in their pockets or positioned within their room. The mobile phone then acts as a base station and transmits the data through the cellular system provided by the mobile service provider to the hospital server. This may be a cloud-based server or a hospital based server.

Chu et al [8] have proposed a ubiquitous personal health surveillance management system is an example of a system which uses a similar architecture using ZigBee technology and a mobile phone. However it is well known that Wi-Fi signals have a detrimental impact on ZigBee networks [10-12]. Furthermore, there has been little investigation of the effect of Bluetooth or a combination of various short range wireless signals on ZigBee or Dash7 signals.

In order to test the effectiveness of these kinds of designs for WRPM systems we looked at the resiliance of these kind of systems to interference effects.

\section{Test Methodology}

Availability comes at the cost of the band being used by an ever growing range of devices which may lead to interference. Interference is very undesirable in a monitoring system where accuracy is vital to the process being tracked.

Bluetooth, ZigBee and Wi-Fi standards operate in the Industrial Scientific and Medical (ISM) bands at a number of shared frequencies as shown in Table I. Although Dash7 uses the ISM band, it utilizes a different frequency range. Therefore, it is expected that its signals would not be significantly affected by the technologies using other parts of the ISM bands.

Consequently we set up an experimental system to test the interference effects between these technologies working in the 2.4GHz band.

TABLE I.

\begin{tabular}{|l|l|c|c|}
\hline \multirow{2}{*}{ Technology } & \multicolumn{3}{|c|}{ Frequencies } \\
\hline Bluetooth & & $2.4 \mathrm{GHz}$ & \\
\hline Wi-Fi & $5 \mathrm{GHZ}(802.11 \mathrm{n})$ & $\begin{array}{c}2.4 \mathrm{GHZ} \\
802.11 \mathrm{~b} / \mathrm{g} / \mathrm{n}\end{array}$ & \\
\hline ZigBee & $868 \mathrm{MHz}$ (Europe) & $\begin{array}{c}915 \mathrm{MHz} \\
\text { (America) }\end{array}$ & $\begin{array}{c}2.4 \mathrm{GHz} \\
\text { (worldwide) }\end{array}$ \\
\hline Dash7 & $433 \mathrm{MHz}$ & & \\
\hline
\end{tabular}

This experiment was conducted to explore the effect of interference on Dash 7 and ZigBee signals. Data packet loss was measured on ZigBee and Dash7 under a range of controlled conditions. The experiment was carried out in a room approximately $10.5 \mathrm{~m} \times 4 \mathrm{~m}$. Table II shows the equipment used while Fig 3 illustrates the position of the devices.

TABLE II.

\begin{tabular}{|l|l|}
\hline \multicolumn{1}{|c|}{ Quantity } & \multicolumn{1}{c|}{ Item } \\
\hline 2 & Wizzikit Dash7 Modules \\
\hline 2 & Xbee ZigBee Modules \\
\hline 1 & Wireless Access Point \\
\hline 1 & Wi-Fi enabled Laptop Computer \\
\hline
\end{tabular}

\begin{tabular}{|l|l|}
\hline \multicolumn{1}{|c|}{ Quantity } & \multicolumn{1}{c|}{ Item } \\
\hline 1 & Desktop Computer \\
\hline 2 & Blutooth Adapter (1 each per computer) \\
\hline
\end{tabular}

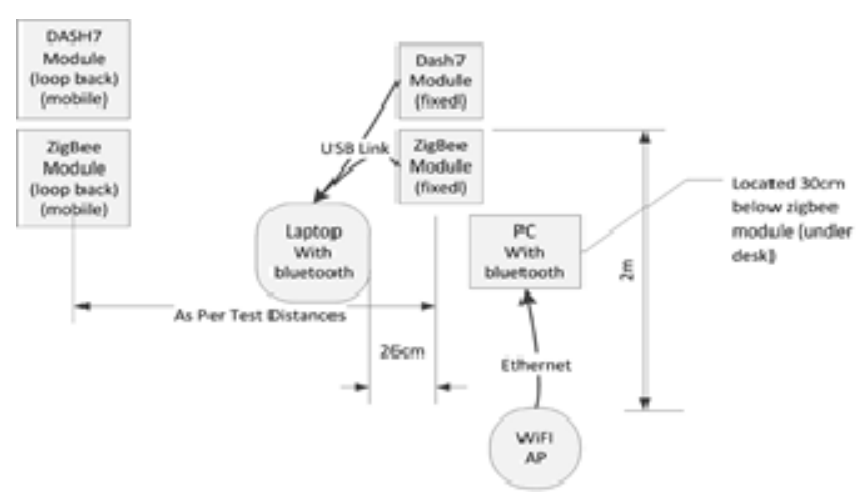

Fig 3. Device Layout

During the experiment, the devices remained in the same position, with the exception of one of the ZigBee and Dash7 modules. These modules were placed as a pair or individually at distance intervals in respect of the stationary devices. Seven distances were used as follows: adjacent, $30 \mathrm{~cm}, 50 \mathrm{~cm}, 1 \mathrm{~m}, 2 \mathrm{~m}$, $5 \mathrm{~m}$ and $10 \mathrm{~m}$. The distances were chosen to represent a range of real-world scenarios. For each position, ten readings were taken, and averaged. This process is repeated for each combination, as illustrated in Table III.

\section{TABLE III.}

\begin{tabular}{cl} 
Test No & \multicolumn{1}{c}{ Device Combination } \\
1 & ZigBee \\
2 & Dash7+Bluetooth \\
3 & Bluetooth+Dash7+ZigBee (Testing ZigBee) \\
4 & Bluetooth+Dash7+ZigBee (Testing Dash7) \\
5 & Bluetooth+Wi-Fi+Dash7 \\
6 & Bluetooth+Wi-Fi+ZigBee \\
7 & Bluetooth+Wi-Fi+ZigBee+Dash7 (Testing ZigBee) \\
8 & Bluetooth+Wi-Fi+ZigBee+Dash7 (Testing Dash7) \\
9 & Wi-Fi+Dash7
\end{tabular}

X-CTU software [13] was used to measure the effects of interference on each signal. This tool sends 100 packets to a remote node which are then sent back to the sender. The rate of packet loss in each round trip is interpreted as indicative of the interference. Before the full scale experiment was conducted a trial run identified some issues regarding power supply and X-CTU's configuration which were rectified before conducting the full scale test.

\section{RESULTS}

We found that whereas ZigBee transmissions were affected by interference, Dash7 transmissions are largely unaffected by the other technologies used in the experiment.

Fig 4 shows the packet loss for Dash7 in each combination. It was found that there was little or no packet loss for this technology and that this was consistent across the full range of distances. It can be seen that there were two packet losses, which were non-reproducible and it is likely that this was due to external signal interferences on the same band as Dash7 (433MHz) however further investigation is required. 


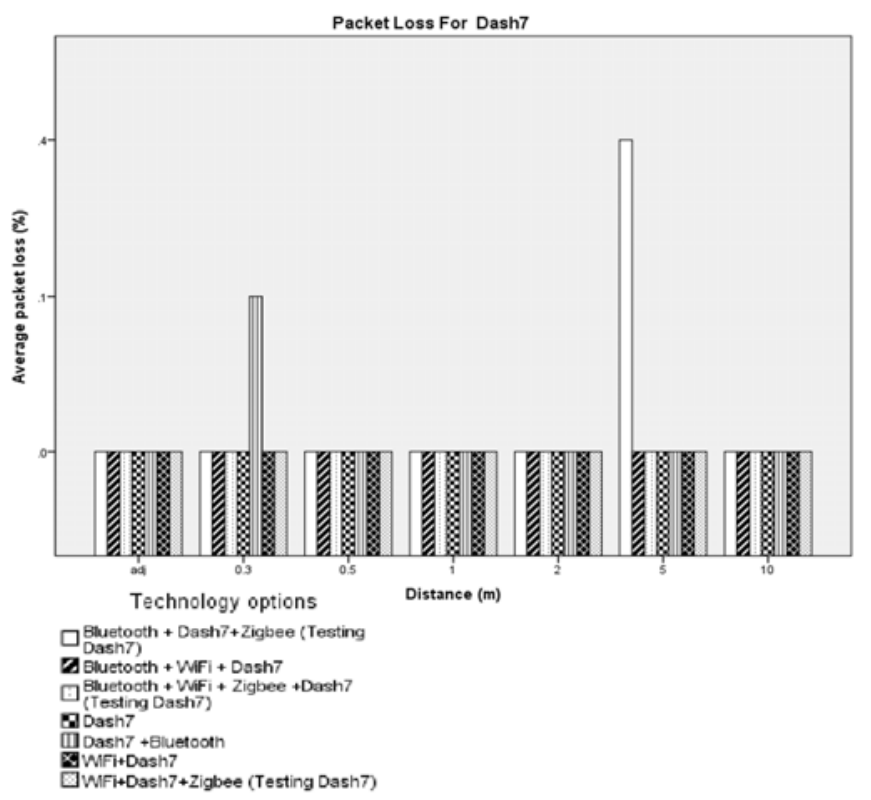

Fig 4. Packet loss for Dash7

The effect of interference on ZigBee signals is much greater and much more significant, as shown in Figure 5. There was no packet loss at distances up-to and including 5m. At the distance of $10 \mathrm{~m}$ there was a small amount of packet loss.

When ZigBee is used in the presence of Bluetooth, there were similar patterns of packet loss, but this was slightly higher at $10 \mathrm{~m}$. However, when Dash7 and Bluetooth were both active, ZigBee suffers more packet losses at the distances of $5 \mathrm{~m}$ and $10 \mathrm{~m}$ as shown in Fig 5.

The effect of Wi-Fi on ZigBee was very severe with the loss of over $80 \%$ of the packets at distances of over $1 \mathrm{~m}$. However there was also a small amount of packet loss at $30 \mathrm{~cm}$. There was no packet loss at the distance of $50 \mathrm{~cm}$ or when the modules were adjacent. This is shown in Fig 5. The unexpected results (no loss at $50 \mathrm{~cm}$ ) could be due to reflections or external interference.

When Dash7 and Wi-Fi were both active, the packet loss of ZigBee increased, compared to the case when just Wi-Fi alone was active. This was true for all the distances, except when they were adjacent. This is shown in Figure 5.

There was also packet loss in respect of ZigBee when both Wi-Fi and Bluetooth modules were active at distances from $30 \mathrm{~cm}$ upwards. With this combination over $40 \%$ of the packets were lost at the distance of $30 \mathrm{~cm}$. Generally, data loss has increased with distance, but at $1 \mathrm{~m}$ and $10 \mathrm{~m}$ the loss is lower. This seems to be unique to this combination of Wi-Fi Bluetooth and ZigBee.

During the experiment, the devices remained in the same position, with the exception of one of the ZigBee and one of the Dash7 modules.

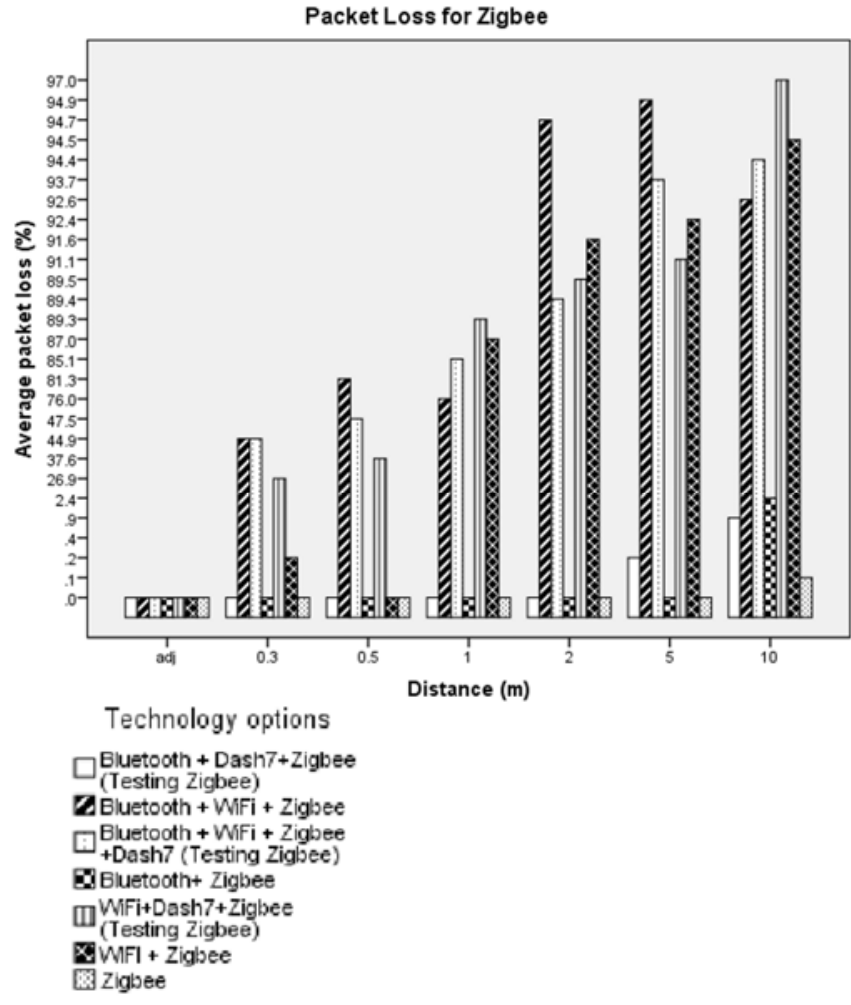

Fig 5. Packet Loss for ZigBee

\section{CONCLUSION}

A wireless remote patient monitoring (WRPM) system based on ZigBee or Dash7 using mobile handsets is proposed. However, the reliability of wireless systems in medical application is central to their effectiveness and any interference with their operation would seriously compromise the monitoring system. We have demonstrated that ZigBee is highly susceptible to signal interference from Wi-Fi and Bluetooth and that Dash7 does not suffer from this problem. ZigBee is highly compromised in a medical environment where reliability of transmission is important. On the other hand Dash7 has proved to be less sensitive to interference and is much more reliable in a medical environment. That is not to say that it is completely immune to possible interference from other communication technologies that operate in the same unlicensed frequency bands. For the present at least, Dash7 appears to be a better choice for wireless patient monitoring systems compared to ZigBee and Bluetooth.

\section{FINAL WORD ON ETHICAL ISSUES}

Wireless remote patient monitoring (WRPM) systems can improve patient comfort and mobility while allowing continuous monitoring. Due to the nature of this technology the network communicates wirelessly without notifying any parties that they or their equipment is being monitored and tracked. Consequently the technology has privacy issues associated with it. [6]. Garfinkel et al. [4] categorizes two threats associated with the use of personal tagging: individual threats and corporate data threats. It is the first of these which is of concern to patients whose data could be intercepted and tracked by third parties.

Kelly and Erickson [7] suggest that tagging objects linked to an individual may be used to collect information about the individual as much as about the object and hence their daily life can come under scrutiny. It may therefore be considered necessary not only to obtain written consent from patients for 
the medical procedures which they undertake. Monitoring through wireless systems may also require additional consent to be given to hospitals in order to use this technology and this may need to become as routine as the request for medical procedure consent.

\section{REFERENCES}

[1] T. Hayajneh, B. J. Mohd, M. Imran, G. Almashaqbeh, and A. V. Vasilakos, "Secure Authentication for Remote Patient Monitoring with Wireless Medical Sensor Networks," Sensors (14248220), vol. 16, pp. 1-25, 2016.

[2] Y. Kim and S. Lee, "Energy-efficient wireless hospital sensor networking for remote patient monitoring," Information Sciences, vol. 282, pp. 332349, 2014.

[3] N. Baisa, "Designing wireless interfaces for patient monitoring equipment," RF DESIGN, vol. 28, pp. 4654, 2005.

[4] R. Sahandi, S. Noroozi, G. Roushan, V. Heaslip, and Y. Liu, "Wireless technology in the evolution of patient monitoring on general hospital wards," Journal of Medical Engineering \& Technology, vol. 34, pp. 51-63, 2010.

[5] J. Y. Khan, M. R. Yuce, and F. Karami, "Performance evaluation of a Wireless Body Area sensor network for remote patient monitoring," Conference Proceedings: ... Annual International
Conference Of The IEEE Engineering In Medicine And Biology Society. IEEE Engineering In Medicine And Biology Society. Annual Conference, vol. 2008, pp. 1266-1269, 2008.

[6] S. Adibi, "Link technologies and BlackBerry mobile health (mHealth) solutions: a review," IEEE Trans Inf Technol Biomed, vol. 16, pp. 586-97, Jul 2012.

[7] M. Arsalan, A. Umair, and V. K. Verma, "Dash7: Performance," IOSR Journal Electronics and Communication Engineering, vol. 2, pp. 08-11.

[8] J. Norair, "Introduction to dash7 technologies," Dash7 Alliance Low Power RF Technical Overview, 2009.

[9] S. Churchill. (2013, 14th April). DASH7 Alliance Announces M2M Standard. Available: http://www.dailywireless.org/2013/09/25/dash7alliance-announces-m2m-standard/

[10] M. U. Memon, L. X. Zhang, and B. Shaikh, "Packet loss ratio evaluation of the impact of interference on zigbee network caused by Wi-Fi (IEEE $802.11 \mathrm{~b} / \mathrm{g}$ ) in e-health environment," 2012, pp. 462-465.

[11] L. Lee, G. Kang, X. Zhang, X. Li, and L. Chen, "An interference avoidance strategy for zigbee based WeHealth monitoring system," 2012, pp. 68-72.

[12] C. J. M. Liang, A. Terzis, N. B. Priyantha, and J. Liu, "Surviving Wi-Fi interference in low power ZigBee networks," 2010, pp. 309-322.

[13] D. International, X-CTU, Digi ed., 2012. 